\title{
The effects of different tensile parameters for the neurodynamic mobilization technique on tricipital muscle wet weight and MuRf-1 expression in rabbits with sciatic nerve injury
}

\author{
Yan Wang ${ }^{1,2^{*}}$, Ming Ma ${ }^{2}$, Qiang Tang ${ }^{1,2}$, Luwen Zhu ${ }^{1,2}$, Melanie Koleini ${ }^{3}$ and Dequan Zou ${ }^{3}$
}

\begin{abstract}
Background: After peripheral nerve injury, muscles without innervation begin to undergo atrophy. Research has suggested that MuRf-1 may play a role in muscle atrophy. The neurodynamic mobilization technique (NMT) is a manual therapy method used to elongate a nerve along its long axis, resulting in improved blood flow to the nerve. However, the nerve can be damaged if elongated too much. The purpose of this study is to observe the effect of NMT on muscle wet weight and MuRf-1 expression in rabbits with sciatic nerve injury.

Methods: Six adult rabbits were measured to determine the relationship between the joint angle of the lower limb and percent of sciatic nerve elongation to define the tensile parameters of NMT; Thirty adult rabbits were randomly assigned into a sham, model, NMT-A, NMT-B, or NMT-C groups. Four weeks post-treatment, the wet mass of the tricipital muscles and MuRf-1 expression were observed.

Results: The wet mass of the tricipital muscles in the NMT-B group was significantly greater than the NMT-A, NMT-C, and model groups. In addition, MuRf-1 expression was significantly reduced in the NMT-B group compared with the NMT-A, NMT-C, and model groups.

Conclusions: Elongating the nerve by NMT of 9\% in rabbits decreased MuRf-1 expression and decelerated muscle atrophy in the subjects with sciatic nerve injury.
\end{abstract}

Keywords: Neurodynamic mobilization technique (NMT), Tricipital muscle wet weight, MuRf-1, Peripheral nerve injury

\section{Introduction}

Nerves are special organs that exist in almost all tissues of the human body [1]. Compared with central nerves, a majority of peripheral nerves are more superficial and lack protection by bony structures, while a few of peripheral nerves are protected by bony structures, such as median never in carpal tunnel, they are more easily compressed than central nerves. Therefore, peripheral nerve injuries are a common clinical problem and often lead to long-term functional deficits [2]. Additionally,

\footnotetext{
* Correspondence: swallow-1113@163.com

${ }^{1}$ Rehabilitation medicine center of the second affiliated hospital of Heilongjiang university of Chinese medicine, Harbin 150001, China ${ }^{2}$ Rehabilitation medicine college of Heilongjiang university of Chinese medicine, Harbin 150040, China

Full list of author information is available at the end of the article
}

after peripheral nerve injury, the muscles without innervations begin to undergo atrophy and even irreversible degeneration. Therefore, the control atrophy and degeneration of denervated muscle has reemerged as a focus of study. MuRf-1 may act by degrading components of the contractile apparatus, its overexpression results in disruption of the muscle [3]. Therefore, inhibition of MuRf-1 may protect against denervated muscle atrophy.

Numerous studies have demonstrated that damaged nerves produce various pathophysiological responses, such as nerve tissue ischemia [4], axonal transport inhibition [5], and intraneural edema [6,7]. The neurodynamic mobilization technique (NMT) is a manual therapy method used by physiotherapists to assess and treat neuromuscular disorders. It includes gliding techniques 
and tensile techniques. Gliding techniques, or 'sliders', attempt to produce a sliding movement between neural structures and adjacent nonneural tissues and is executed in a non-provocative fashion. As the name implies, the purpose of neurodynamic tensile techniques are more aggressive than neurodynamic 'sliders'. NMT is used to increase axonal transport and improve nerve conduction $[8,9]$, and reduce the pressure existing within the nerve, thereby resulting in improved blood flow to the nerve. This increased flow may promote the regeneration and healing of the injured nerve [10]. A study also suggested that axon stretching may speed the rate of slow axonal transport and neuron growth [11]. Nerves possess viscoelastic properties $[12,13]$, the reduction in stress is most rapid early in elongation. As a peripheral nerve elongates, its blood flow changes correspondingly. Clark et al. reported that nerve blood flow decreased approximately $50 \%$ when the nerve was elongated approximately $8 \%$ with substantial recovery, whereas an approximate $80 \%$ reduction in blood flow was observed after $15 \%$ elongation with minimal recovery. In addition, mechanical failure of the restored nerve was noted after 16 to $17 \%$ elongation [14]. Therefore, defining the tensile parameters of NMT is a key point in this study.

The purpose of this study is to determine the relationship between the lower limb joining angle and sciatic nerve elongation to define the tensile parameters of NMT and to observe the effect of NMT on tricipital muscle wet weight and MuRf- 1 expression in rabbits with sciatic nerve injury. In view of the effects of NMT, we hypotheses that NMT decelerate muscle atrophy by restraining MuRf-1 expression.

\section{Material and methods}

All experiments in this study were performed in compliance with the guidelines of the Second Affiliated Hospital of Heilongjiang University of Chinese medicine Institutional Laboratory Animal Care and Use Committee (ABX20130601A).

\section{To determine the relationship between the lower limb} joint angle and sciatic nerve elongation to define the tensile parameters of NMT

Six 4 5-months old male adult Japanese white rabbits with a mean weight of $1.79 \mathrm{~kg}$ were supplied by Harbin Pacific Biological Pharmaceutical Co., LTD. [license SCXK (Hei), 2011-009]. After randomly numbering the rabbits from 1 to 6 , they were anaesthetized with 1$2 \mathrm{ml} / \mathrm{kg}$ of $10 \%$ chloral hydrate via the ear marginal vein. A lateral approach was created, the attachment point of rectus femoris tendon on knee joint and the rectus femoris between up one third and middle were cut, and the sciatic nerve was exposed fully. Next, two surgical sutures, one was on $1 \mathrm{~cm}$ above lateral femoral epicondyle, another was on going up $20 \mathrm{~mm}$ apart, were fastened to the nerve trunk as markers. The rabbits were placed on the experiment table with their hip and knee joints at $45^{\circ}$ and $30^{\circ}$ angles, respectively. The knee joints of the rabbits were passively extended along the sciatic nerve. Meanwhile, Digital Vernier Calipers were placed on the nerve trunk with a precision of $0.01 \mathrm{~mm}$. When the distance between the two markers in the nerve trunk was elongated to the appropriate length (nerve elongation 6\%, $9 \%$ and $12 \%$ is $21.2 \mathrm{~mm}, 21.8 \mathrm{~mm}$ and $22.4 \mathrm{~mm}$, respectively), the angle of the knee joint was measured. The mean of the knee joint angles of the six rabbits was used as the standard of NMT for different nerve elongations (Figures 1,2, and 3). The relationship between the lower limb joint angle and sciatic nerve elongation is described as Table 1.

\section{The effect of NMT on muscle atrophy and MuRf-1 expression}

Thirty 4 5-months old male adult Japanese white rabbits with a mean weight of $1.81 \mathrm{~kg}$ were supplied by the same company. Animals were randomly assigned to the sham group $(n=6)$, model group $(n=6)$, NMT-A group (nerve elongation of $6 \%, \mathrm{n}=6$ ), NMT-B group (nerve elongation of $9 \%, n=6$ ), and NMT-C group (nerve elongation of $12 \%, n=6$ ). The rabbits were anaesthetized, and the sciatic nerves were exposed as described above. The sham group was subjected to the same surgical procedure with sciatic nerve exposure but without crush injury. The hemostatic forceps was covered by an infusion set and clamped to the nerve trunk $(1.5 \mathrm{~cm}$ above the lateral femoral epicondyle) with 3 buttons for 5 minutes; this caused approximately $2 \mathrm{~mm}$ of damage to the nerve.

The intervention methods for all groups as follows: sham and model groups: no intervention; NMT groups: based on the above experimental methods, intervention by NMT was conducted on the rabbits in the 3 groups 3 days after the operation. Before treatment, each rabbit was placed on the experiment table and with the lesioned limb hanging off the side of the table. Then the rabbit's hip joint was flexed to $45^{\circ}$ and the knee joint flexed to $30^{\circ}$. The knee joint was extended from $30^{\circ}$ to $90^{\circ}$ (NMT-A), $30^{\circ}$ to $130^{\circ}$ (NMT-B), or $30^{\circ}$ to $170^{\circ}$ (NMT-C). The position of the limb was marked for each condition, as line 1,2,3 respectively. For rabbits in the NMT-A treatment group, a wooden blade was put on line 1 , the researcher quickly extended the knee joint from start angle to line 1 and, then back to the start angle while an assistant stabilized the rabbit on the table. Rabbits in the NMT-B and NMT-C treatment groups received the same treatment as the NMT-A 


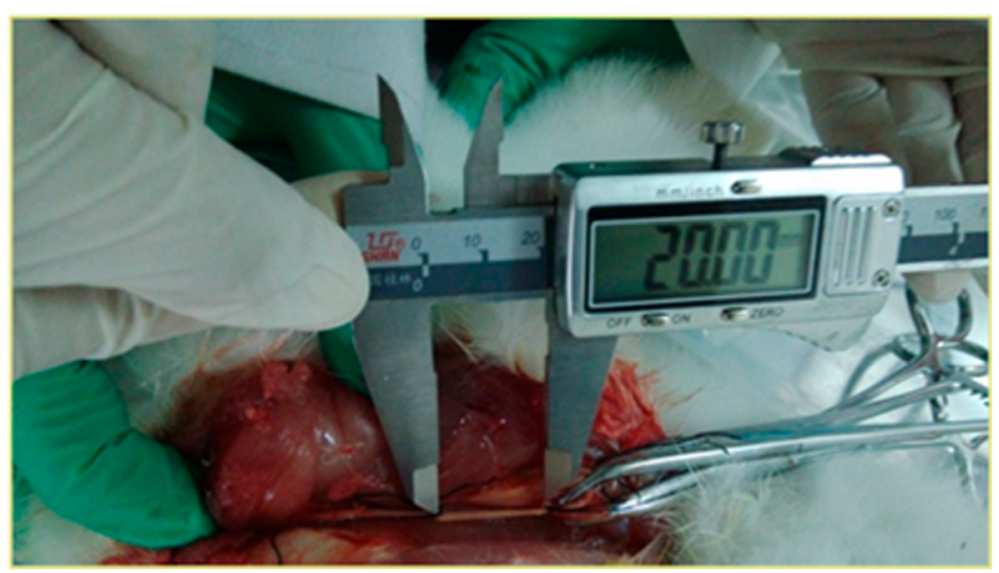

Figure 1 Original length of the sciatic nerve $(\mathrm{mm})$.

group, except a wooden blade was placed on line 2 or 3 respectively. This NMT methodology produce 'sliders' which is non-provocative, because the damaged nerves are more mechanosensitive than healthy nerves. Each operation time was $1 \mathrm{~s}$, and each relaxation time was $5 \mathrm{~s}$; both were performed 10 times/group, 1group/day, and 6 days/week for a total treatment cycle duration of 4 weeks. At 4 weeks post-treatment, the bilateral tricipital muscles of the rabbits were excised. First, the tendons of gastrocnemius attached at medial and lateral condyles of femur were severed. Then the Achilles tendon was also cut. Next, the entire tricipital muscle was excised from the soft tissues connected with tibia and fibula. Finally, of the remaining soft tissue on the tricipital muscle was removed. The muscle wet mass was weighed using an electronic analytical balance with a precision of $0.1 \mathrm{~g}$. Then, MuRf- 1 expression in the triceps was measured by Western blot. Samples $(200 \mu \mathrm{g})$ of skeletal muscle were ground and dissolved in the cold cracking protein liquid and centrifuged $(12000 \mathrm{r} / \mathrm{min}$, $10 \mathrm{~min})$. A sample of the liquid mixed with protein $(40 \mu \mathrm{g})$ was boiled (5 min). After electrophoresis, proteins were transferred to a nitrocellulose membrane, blocked with $1 \%$ nonfat milk, and incubated overnight at $4^{\circ} \mathrm{C}$. The membranes were then washed with large volumes of TBST (3 times, for $10 \mathrm{~min}$ each), and exposed to a primary antibody overnight at $4^{\circ} \mathrm{C}$. The membranes were then washed again ( 3 times, for $10 \mathrm{~min}$ each), then incubated with second antibody for $2 \mathrm{~h}$ at $37^{\circ} \mathrm{C}$. Membranes were covered with $\mathrm{ECL}^{\mathrm{mix}}$ detection reagents and imaged (autoradiography film). The resulting bands on the films were analyzed to determine protein levels.

Statistical calculations were performed using SPSS 19.0 for Windows. The data of wet mass of muscles and MuRf-1 protein expression in this article were presented

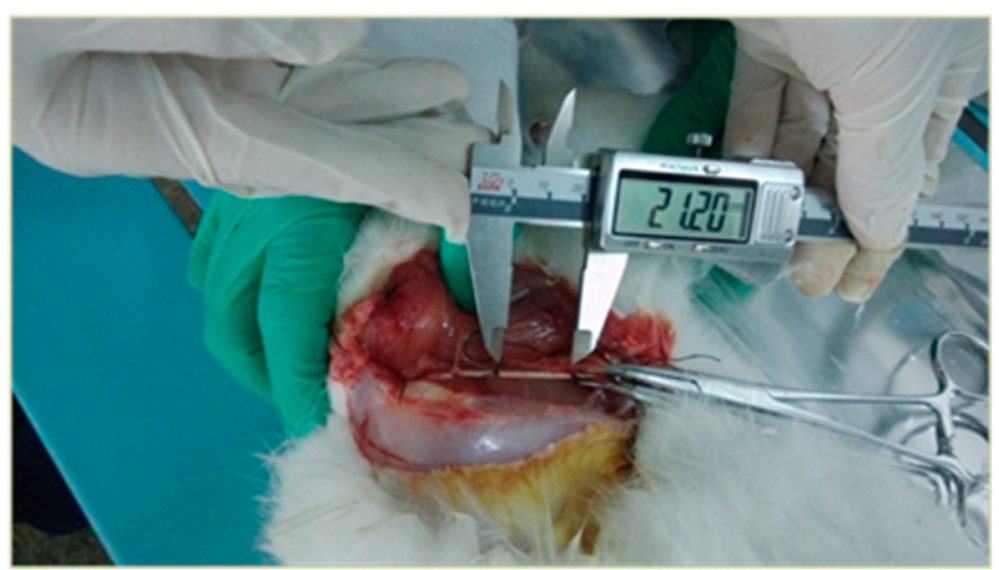

Figure 2 The sciatic nerve elongated $6 \%(\mathrm{~mm})$. 


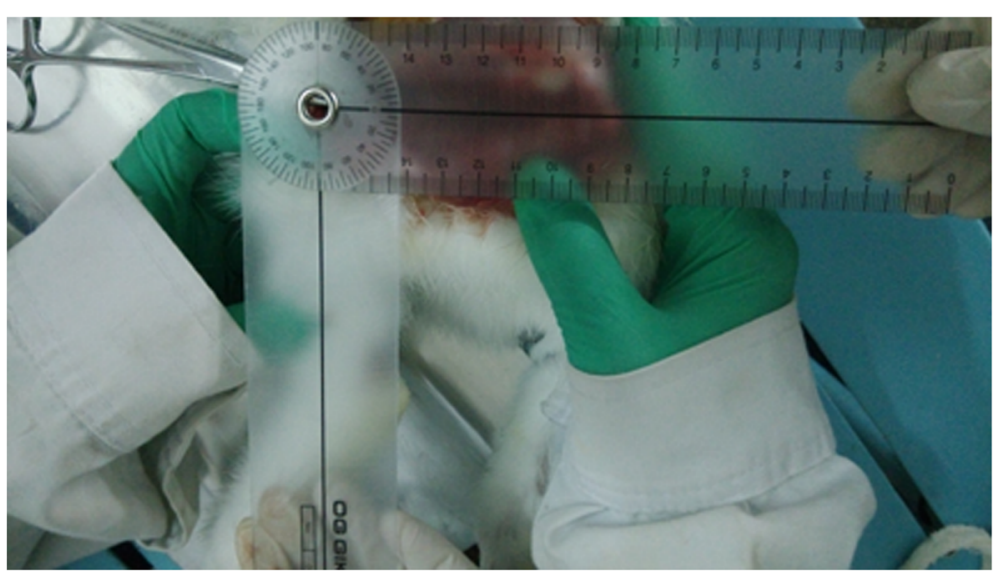

Figure 3 The knee joint angle of the sciatic nerve elongated by $6 \%$.

as means $\pm \mathrm{SD}$. All variables were tested for normal distribution using the Kolmogorov-Smirnov test ( $p>0.05$ ). One-way analysis of variance (ANOVA) followed by the LSD post-hoc test was used for the statistical analysis. A significant difference was defined as $\mathrm{p} \leq 0.05$.

\section{Results}

\section{Wet mass of the tricipital muscles}

The wet mass of the tricipital muscle was significantly less in the lesioned lateral muscle compared with the normal lateral muscle in all groups $(\mathrm{p}<0.05)$ except the sham group $(p=0.289)$. Further, the tricipital muscle wet mass in the NMT-A and NMT-B groups was significantly increased compared to the model group $(\mathrm{p}<0.05)$, whereas no significant difference was noted between the model and NMT-C groups $(\mathrm{p}>0.05)$. The tricipital muscle wet mass in the NMT-B group was significantly higher than the NMT-A and NMT-C groups (Table 2 and Figure 4).

\section{MuRf-1 protein analyses}

MuRf-1 protein expression increased in the lesioned lateral muscle compared with the normal lateral muscle in all groups $(\mathrm{p}<0.05)$ except in the sham group $(\mathrm{p}=0.197)$. In the lesioned lateral muscle, MuRf-1 protein expression was lower in the NMT-A and NMT-B groups compared to the model group $(\mathrm{p}<0.05)$;

Table 1 Relationship between the knee angle and sciatic nerve elongation

\begin{tabular}{llllllll}
\hline Nerve elongation & No 1 & No 2 & No 3 & No 4 & No 5 & No 6 & Mean \\
\hline $6 \%$ & $92^{\circ}$ & $90^{\circ}$ & $89^{\circ}$ & $90^{\circ}$ & $90^{\circ}$ & $90^{\circ}$ & $90.1^{\circ}$ \\
$9 \%$ & $128^{\circ}$ & $135^{\circ}$ & $130^{\circ}$ & $133^{\circ}$ & $126^{\circ}$ & $128^{\circ}$ & $130^{\circ}$ \\
$12 \%$ & $168^{\circ}$ & $174^{\circ}$ & $170^{\circ}$ & $169^{\circ}$ & $170^{\circ}$ & $168^{\circ}$ & $169.8^{\circ}$ \\
\hline
\end{tabular}

whereas no significant difference was observed between the model and NMT-C groups ( $\mathrm{p}>0.05)$. MuRf- 1 protein expression was reduced in the NMT-B group compared to the NMT-A and NMT-C groups $(\mathrm{p}<0.05)$ (Table 3 and Figures 5 and 6).

These results indicate that NMT performed within the scope of safety may effectively reduce MuRf- 1 protein expression and reduce muscle atrophy after nerve injury. Western blotting results indicate that the levels of MuRf-1 in the NMT-B group were reduced compared with other groups, except the sham group.

\section{Discussion}

Previous studies of NMT have focused on neuropathic pain both in clinical trials and animal experiments [14-17]. Although these studies provide valuable information regarding peripheral nerve movement and strain, they provide no insight into the mechanisms responsible for the benefits of using NMT on denervated muscle. Moreover, the tensile parameters of NMT were not

Table 2 Comparisons of the wet mass of bilateral tricipital muscles $(g,-X \pm s)$

\begin{tabular}{lll}
\hline Group & Lesion lateral & Normal lateral \\
\hline Sham group $(n=6)$ & $6.57 \pm 0.08$ & $6.65 \pm 0.14$ \\
Model group $(n=6)$ & $2.48 \pm 0.13^{*}$ & $6.73 \pm 0.08$ \\
NMT-A group $(n=6)$ & $3.27 \pm 0.10 \#$ & $6.67 \pm 0.10$ \\
NMT-B group $(n=6)$ & $4.07 \pm 0.12 \boldsymbol{\Delta}$ & $6.72 \pm 0.10$ \\
NMT-C group $(n=6)$ & $2.52 \pm 0.10 \succsim$ & $6.65 \pm 0.10$ \\
\hline
\end{tabular}

Lesioned lateral compared with normal lateral muscle, ${ }^{*} \# \mathbf{\Delta}$ is $\mathrm{p}<0.05$ in the model, NMT-B, NMT-A, and NMT-C group, respectively. In the lesioned lateral, the wet mass of muscle in the model, NMT-B, NMT-A, and NMT-C group compared with in the sham group, *\# $\boldsymbol{\Delta}$ 站 $\mathrm{p}<0.05$; wet mass of muscle in the NMT-B and NMT-A compared with in the model group, $\# \mathbf{\Delta} p<0.05$; the wet mass of muscle in the NMT-B compared with in the NMT-A and NMT-C group, \# ts $\mathrm{p}<0.05$. 




Figure 4 Wet mass of bilateral tricipital muscles. A significant reduction in the tricipital muscle wet mass was noted in the lesioned lateral muscle compared with the normal lateral muscle in all groups $(p<0.05)$ except the sham group $(p>0.05)$.In the lesioned lateral, the tricipital muscle wet mass in the NMT-B group was significantly increased compared with the NMT-A, NMT-C and model groups ( $p<0.05)$.

regulated in animal experiments. Driscoll et al. reported that nerve blood flow is reduced by approximately $70 \%$ and $78 \%$ after $8.8 \%$ and $16.1 \%$ strain, respectively; however, upon release of traction, blood flow exceeded baseline recordings, increasing to a mean of $151 \%$ of baseline blood flow after $8.8 \%$ strain and $82 \%$ of baseline after $16.1 \%$ strain [12]. Others studies have also concluded that peripheral nerve elongation of less than $10 \%$ is safe and feasible [18]. Therefore, we chose a median elongation of $9 \%$, and fluctuated down and up $3 \%$ to an elongation of $6 \%$ and $12 \%$, respectively, to observe the influence of NMT on atrophy of denervated muscle. In this study, we report the relationship between the lower limb joint angle and sciatic nerve elongation. We also found different effects of NMT on muscle wet weight and MuRf-1 expression in rabbits with sciatic nerve injury.

Table 3 Comparison of MuRf-1 protein expression in bilateral muscles $(-\mathrm{X} \pm \mathrm{s})$

\begin{tabular}{lll}
\hline Group & Lesion lateral & Normal lateral \\
\hline Sham group $(n=6)$ & $0.05 \pm 0.00$ & $0.050 \pm 0.000$ \\
Model group $(n=6)$ & $1.04 \pm 0.05^{*}$ & $0.052 \pm 0.000$ \\
NMT-A group $(n=6)$ & $0.67 \pm 0.07 \#$ & $0.051 \pm 0.000$ \\
NMT-B group $(n=6)$ & $0.43 \pm 0.04 \boldsymbol{\Delta}$ & $0.051 \pm 0.000$ \\
NMT-C group $(n=6)$ & $1.01 \pm 0.03 \star$ & $0.050 \pm 0.000$ \\
\hline
\end{tabular}

Lesioned lateral compared with normal lateral muscle, *\# $\boldsymbol{\Delta} \sim \mathrm{s} p 0.05$ in the model, NMT-B, NMT-A, and NMT-C group, respectively.

In the lesioned lateral, the MuRf- 1 protein expression in the model, NMT-B, NMT-A, and NMT-C group compared with in the sham group, ${ }^{*} \boldsymbol{\Delta}$. $\mathrm{p}<0.05$; the MuRf-1 protein expression in the NMT-B and NMT-A compared with in the model group, $\# \mathbf{\Delta} p<0.05$; the MuRf-1 protein expression in the NMT-B compared with in the NMT-A and NMT-C group, \# $\mathrm{p}<0.05$.
Mild peripheral nerve injury may result in epineurial edema; with compression that is prolonged or of significant magnitude, the resulting endoneurial edema subsequently leads to fibrosis and adhesions [19]. Even worse, denervated muscle gradually atrophies and loses function. The pathogenesis of atrophy of denervated muscle is unclear. Nagata et al. suggested that a prolonged reinnervation process leads to skeletal muscle atrophy from disuse over time [20]. Fortunately, unlike the central nervous system (CNS), the peripheral nervous system (PNS) has an innate capacity to regenerate itself by innervating the muscle again [21]. Additionally, oxygen and nutrients inside the peripheral nerves are associated with nerve regeneration. However, vessels of peripheral nerve distribute particularly. Large blood vessels are primarily observed running longitudinally in the epineurial tissue, and branches of wide capillaries are present within the endoneurial compartment. NMT in the nervous system affects the availability of oxygen and nutrients as well as the ability to dispose of waste products, both of which are critical to nerve regeneration. Brown et al. found that the 'pumping' action that occurs with NMT may disperse the intraneural fluid and result in an altered intraneural pressure [22]. The above studies all demonstrated that NMT may improve nerve regeneration. Santiago et al. observed some reduction in muscle atrophy in rats that received an autograft and guide with adipose precursor cells compared with rats with untreated defects [23]. This finding suggested that nerve regeneration reduces muscle atrophy. Our results also indicate that in lateral lesions, the wet mass of the tricipital muscles in NMT-B group (9\%) increased compared with the other groups. Whereas, the wet mass of the tricipital muscles in NMT-C (12\%) decreased. These 


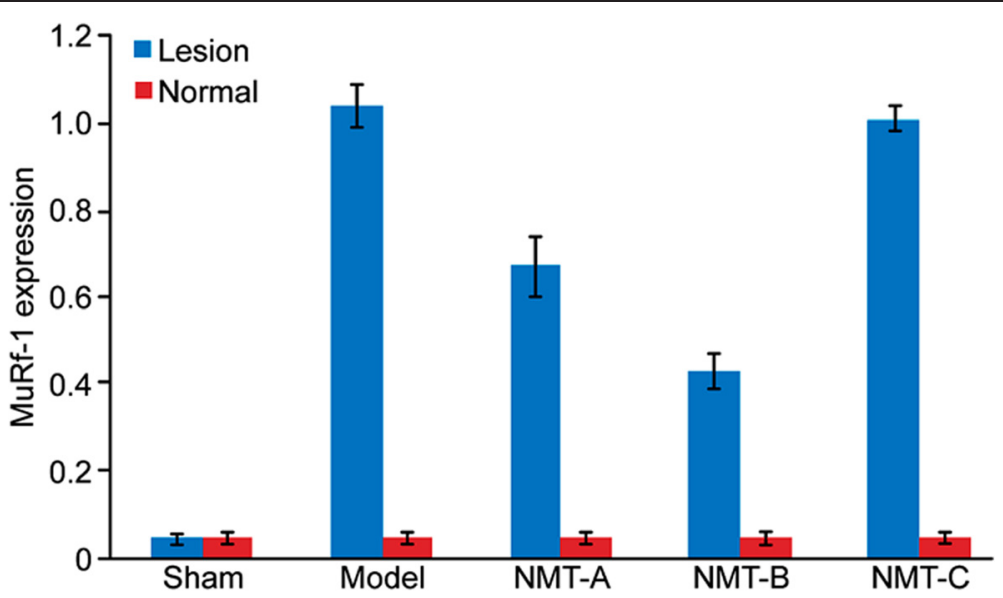

Figure 5 MuRf-1 expression in bilateral muscles. A significant increase in MuRf-1 protein expression was noted in the lesioned lateral muscle compared with the normal lateral muscle in all groups $(p<0.05)$ except the sham group $(p>0.05)$. In the lesioned lateral muscle, significantly reduced MuRf-1 protein expression was noted in the NMT-B group compared with the NMT-A, NMT-C and model groups.

results are similar to those in the study by Driscoll et al. that indicated that nerve blood flow already exceeded baseline recordings after $8.8 \%$ strain and reduced after $16.1 \%$ strain when traction was released [12]. Therefore, we believe that NMT of nerves elongated by approximately $9 \%$ potentially aids in nerve regeneration and reduces muscle atrophy, whereas elongation greater than $12 \%$ is potentially harmful to the nerve. In vivo studies in a MuRf-1deficient mouse model demonstrated that limb muscle degeneration after a sciatic nerve lesion was decelerated by $36 \%$ [24]. MuRf- 1 expression is negatively correlated to the degree of muscle atrophy. The Western blot results in our study indicated that MuRf-1 expression in the NMT-B group (9\%) was lower than in all other groups except the sham group. Gumucio et al. reported the modulation of its expression via physical activity has the potential to prevent or reverse muscle atrophy [25]. Similarly, this study demonstrated that NMT resulting in nerve elongation of approximately $9 \%$ is an effective intervention method to decreased MuRf-1 expression, thereby decelerating muscle atrophy.

\section{Conclusions}

Taken all together, our study for the first time defined the tensile parameters of NMT and demonstrated that elongating the nerve by NMT of approximately $9 \%$ in rabbits decreases MuRf-1 expression and decelerates muscle atrophy in the subjects with sciatic nerve injury. However, the current results are limited by the absence of direct evidence regarding the path by which NMT reduces MuRf- 1 expression and decelerates muscle atrophy. Further studies will be required to identify the mechanism by which NMT reverses denervation muscle atrophy via signaling pathways.

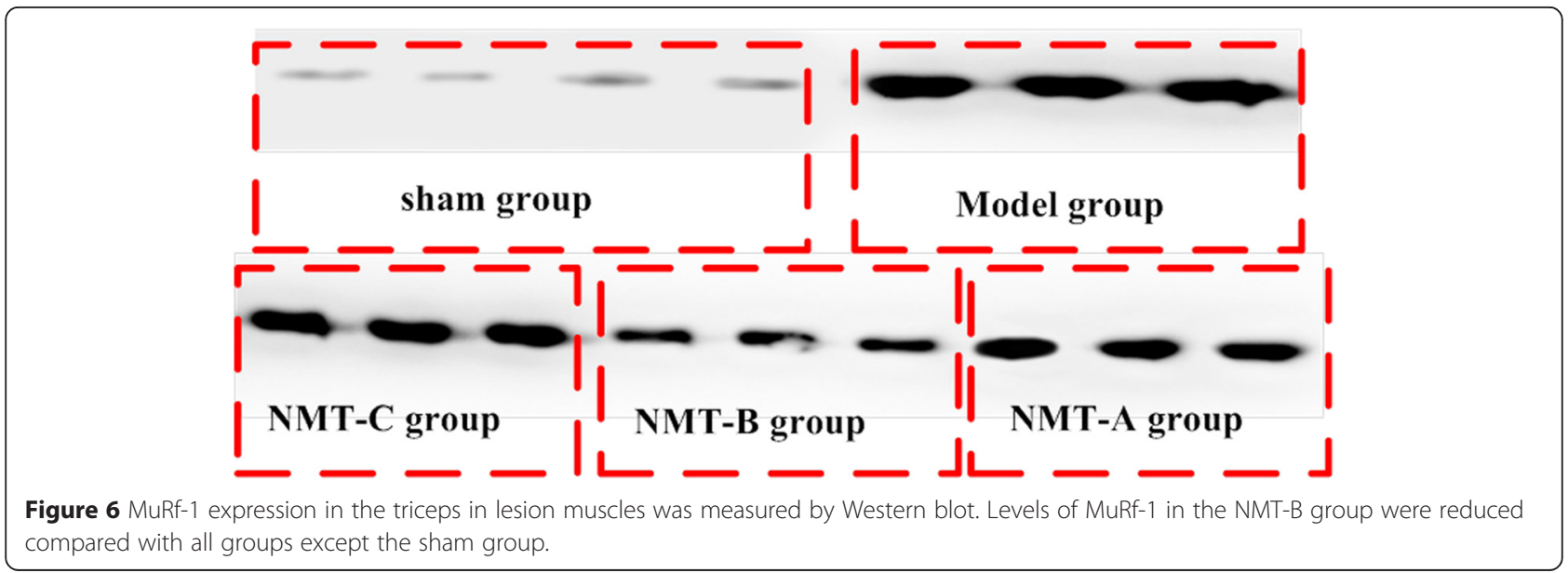




\section{Competing interests}

The authors declare that they have no competing interests.

\section{Authors' contributions}

YW initiated the project, designed the experiments, analyzed the results and wrote the paper, with input from all the authors. MM, QT and LZ carried out the experiment, MK and DZ modified the paper. All authors reviewed and approved the final manuscript.

\section{Acknowledgments}

This study was supported by the Fund of Innovative Talents of the Heilongjiang University of Chinese Medicine (grant number 051290). Additional funding for this study was provided by a postdoctoral research award from the Administration Office of Heilongjiang Postdoctoral Program (grant number LBH-Z13202). We also thank Dr. Zhongping Xu in Surgical Department of Washington University School of Medicine and all our study participants.

\section{Author details}

${ }^{1}$ Rehabilitation medicine center of the second affiliated hospital of Heilongjiang university of Chinese medicine, Harbin 150001, China. ${ }^{2}$ Rehabilitation medicine college of Heilongjiang university of Chinese medicine, Harbin 150040, China. ${ }^{3}$ Program in Physical Therapy, Washington University School of Medicine, St. Louis, MO 63110, USA.

Received: 16 October 2014 Accepted: 10 April 2015

\section{Published online: 15 April 2015}

\section{References}

1. Geuna S, Raimondo S, Ronchi G, Di Scipio F, Tos P, Czaja K, et al. Chapter 3: histology of the peripheral nerve and changes occurring during nerve regeneration. Int Rev Neurobiol. 2009;87:27-46.

2. Jung $Y, \mathrm{Ng} \mathrm{JH}$, Keating $\mathrm{CP}$, Senthil-Kumar P, Zhao J, Randolph MA, et al. Comprehensive evaluation of peripheral nerve regeneration in the acute healing phase using tissue clearing and optical microscopy in a rodent model. PLoS One. 2014;9:e94054.

3. Pizon V, lakovenko A, Van Der Ven PF, Kelly R, Fatu C, Furst DO, et al Transient association of titin and myosin with microtubules in nascent myofibrils directed by the MURF2 RING-finger protein. J Cell Sci. 2002;115:4469-82.

4. Lundborg G. Structure and function of the intraneural microvessels as related to trauma, edema formation, and nerve function. J Bone Joint Surg Am. 1975;57:938-48.

5. Yan JG, Matloub HS, Sanger JR, Zhang LL, Riley DA. Vibration-induced disruption of retrograde axoplasmic transport in peripheral nerve. Muscle Nerve. 2005;32:521-6.

6. Dahlin LB, Archer DR, McLean WG. Axonal transport and morphological changes following nerve compression. An experimental study in the rabbit vagus nerve. J Hand Surg (Br). 1993;18:106-10.

7. Rempel D, Dahlin L, Lundborg G. Pathophysiology of nerve compression syndromes: response of peripheral nerves to loading. J Bone Joint Surg Am. 1999;81:1600-10.

8. Shacklock M. Response to Butler and Coppieters 2007, letter to the editor: clinical neurodynamics-throwing the baby out with the bath water. Man Ther. 2009;14:e1-2.

9. Butler DS. Adverse mechanical tension in the nervous system: a model for assessment and treatment. Aust J Physiother. 1989;35:227-38.

10. Ellis RF, Hing WA. Neural mobilization: a systematic review of randomized controlled trials with an analysis of therapeutic efficacy. J Man Manip Ther. 2008;16:8-22.

11. Eschbach J, Dupuis L. Cytoplasmic dynein in neurodegeneration. Pharmacol Ther. 2011;130:348-63.

12. Driscoll PJ, Glasby MA, Lawson GM. An in vivo study of peripheral nerves in continuity: biomechanical and physiological responses to elongation. J Orthop Res. 2002;20:370-5.

13. Kwan MK, Wall EJ, Massie J, Garfin SR. Strain, stress and stretch of peripheral nerve. Rabbit experiments in vitro and in vivo. Acta Orthop Scand. 1992;63:267-72.

14. Clark WL, Trumble TE, Swiontkowski MF, Tencer AF. Nerve tension and blood flow in a rat model of immediate and delayed repairs. J Hand Surg [Am]. 1992;17:677-87.
15. Santos FM, Silva JT, Giardini AC, Rocha PA, Achermann AP, Alves AS, et al. Neural mobilization reverses behavioral and cellular changes that characterize neuropathic pain in rats. Mol Pain. 2012;8:57.

16. Heebner ML, Roddey TS. The effects of neural mobilization in addition to standard care in persons with carpal tunnel syndrome from a community hospital. J Hand Ther. 2008;21:229-40. quiz 241.

17. Bakhtiary AH, Rashidy-Pour A. Ultrasound and laser therapy in the treatment of carpal tunnel syndrome. Aust J Physiother. 2004;50:147-51.

18. Millesi H, Zoch G, Reihsner R. Mechanical properties of peripheral nerves. Clin Orthop Relat Res. 1995;314:76-83.

19. Rydevik B, Lundborg G. Permeability of intraneural microvessels and perineurium following acute, graded experimental nerve compression. Scand J Plast Reconstr Surg. 1977;11:179-87.

20. Nagata K, Itaka K, Baba M, Uchida S, Ishii T, Kataoka K. Muscle-targeted hydrodynamic gene introduction of insulin-like growth factor-1 using polyplex nanomicelle to treat peripheral nerve injury. J Control Release. 2014;183:27-34.

21. Cerri F, Salvatore L, Memon D, Martinelli Boneschi F, Madaghiele M, Brambilla $P$, et al. Peripheral nerve morphogenesis induced by scaffold micropatterning. Biomaterials. 2014;35:4035-45.

22. Brown CL, Gilbert KK, Brismee JM, Sizer PS, Roger James C, Smith MP. The effects of neurodynamic mobilization on fluid dispersion within the tibial nerve at the ankle: an unembalmed cadaveric study. J Man Manip Ther. 2011;19:26-34

23. Santiago LY, Clavijo-Alvarez J, Brayfield C, Rubin JP, Marra KG. Delivery of adipose-derived precursor cells for peripheral nerve repair. Cell Transplant. 2009;18:145-58.

24. Bodine SC, Latres E, Baumhueter S, Lai VK, Nunez L, Clarke BA, et al. Identification of ubiquitin ligases required for skeletal muscle atrophy. Science. 2001;294:1704-8.

25. Gumucio JP, Mendias CL. Atrogin-1, MuRF-1, and sarcopenia. Endocrine. 2013;43:12-21.

\section{Submit your next manuscript to BioMed Central and take full advantage of:}

- Convenient online submission

- Thorough peer review

- No space constraints or color figure charges

- Immediate publication on acceptance

- Inclusion in PubMed, CAS, Scopus and Google Scholar

- Research which is freely available for redistribution 